\title{
Design Automation and Test Solutions for Digital Microfluidic Biochips
}

\author{
Krishnendu Chakrabarty, Fellow, IEEE
}

(Invited Tutorial)

\begin{abstract}
Microfluidics-based biochips are revolutionizing high-throughput sequencing, parallel immunoassays, blood chemistry for clinical diagnostics, and drug discovery. These devices enable the precise control of nanoliter volumes of biochemical samples and reagents. They combine electronics with biology, and they integrate various bioassay operations, such as sample preparation, analysis, separation, and detection. Compared to conventional laboratory procedures, which are cumbersome and expensive, miniaturized biochips offer the advantages of higher sensitivity, lower cost due to smaller sample and reagent volumes, system integration, and less likelihood of human error. This tutorial paper provides an overview of droplet-based "digital" microfluidic biochips. It describes emerging computer-aided design (CAD) tools for the automated synthesis and optimization of biochips from bioassay protocols. Recent advances in fluidic-operation scheduling, module placement, droplet routing, pin-constrained chip design, and testing are presented. These CAD techniques allow biochip users to concentrate on the development of nanoscale bioassays, leaving chip optimization and implementation details to design-automation tools.
\end{abstract}

Index Terms-Chip layout, computer-aided design (CAD), droplet routing, lab-on-chip, synthesis, testing and diagnosis.

\section{INTRODUCTION}

A DVANCES in digital microfluidics have led to the promise of miniaturized biochips for applications such as immunoassays for point-of-care medical diagnostics, DNA sequencing, and the detection of airborne particulate matter [1]-[8]. These devices enable the precise control of nanoliter droplets of biochemical samples and reagents, and integrated circuit (IC) technology can be used to transport and process "biochemical payload" in the form of tiny droplets. Biochips facilitate the convergence of electronics with the life sciences, and they integrate on-chip various bioassay operations, such as sample preparation, analysis, separation, and detection [1], [4]. Compared to conventional laboratory procedures, which are cumbersome and expensive, miniaturized biochips offer the advantages of higher sensitivity, lower cost due to smaller sample

Manuscript received September 25, 2009; revised November 21, 2009. First published December 31, 2009; current version published January 13, 2010. This work was supported in part by the National Science Foundation under Grant IIS0312352, Grant CCF-0541055, and Grant CCF-0914895, and by the National Institute of General Medical Sciences of the National Institute of Health under Grant R44GM072155. this paper was recommended by Associate Editor W. A. Serdijn.

The author is with the Department of Electrical and Computer Engineering, Duke University, Durham, NC 27708, USA (e-mail: krish@ee.duke.edu).

Digital Object Identifier 10.1109/TCSI.2009.2038976 and reagent volumes, higher levels of system integration, and less likelihood of human error. As a result, non-traditional biomedical applications and markets are opening up fundamentally new uses for ICs. For example, the worldwide market for in vitro diagnostics in 2007 was estimated at $\$ 38$ billion [70], and 1.5 billion diagnostic tests/year worldwide has been predicted for malaria alone [71].

However, continued growth in this emerging field depends on advances in chip/system integration. In particular, design methods are needed to ensure that biochips are as versatile as the macro-labs that they are intended to replace. The few commercial biochips available today (e.g., from Agilent, Fluidigm, Caliper, I-Stat, BioSite, etc.) are specific to an application and they offer no flexibility to the user. Intel recently announced the Health Guide PHS6000 product for home patients, but the underlying technology does not exploit the benefits of reconfigurable microfluidics.

This tutorial paper is focused on droplet-based "digital" microfluidic biochips. The digital microfluidics platform offers the flexibility of dynamic reconfigurability and software-based control of multifunctional biochips. Next the paper describes emerging computer-aided design (CAD) tools for the automated synthesis and optimization of biochips from bioassay protocols. Recent advances on fluidic-operation scheduling, module placement, droplet routing, testing, and dynamic reconfiguration are also presented. These CAD techniques allow biochip users to concentrate on the development of nanoscale bioassays, leaving chip optimization and implementation details to design-automation tools.

It is expected that an automated design flow will transform biochip research and use, in the same way as design automation revolutionized IC design in the 1980s and 1990s. This approach is, therefore, especially aligned with the vision of functional diversification and "More than Moore", as articulated in the International Technology Roadmap for Semiconductors (ITRS) 2007, which highlights "Medical" as being a "System Driver" for the future [9]. Biochip users will adapt more easily to emerging technology if appropriate design methods/tools and in-system automation methods are available. A limitation of current $\mathrm{CAD}$ techniques is that they do not adequately consider unique constraints that arise due to the fluidic aspects of the underlying technology, the likelihood of cross-contamination between different bio-molecules, and the limited availability of stock solutions for use in assay protocols in biochemistry.

The rest of this paper is organized as follows. Section II describes biochip technology platforms, including digital microfluidics. Section III presents synthesis techniques, including 
solutions published in the literature for operation scheduling, module placement, and droplet routing. Section IV describes pin-constrained chip methods. Section V presents advances in testing, diagnosis, and dynamic reconfiguration. Finally, Section VI concludes the paper.

\section{TeChNOlOGy PlatForms}

Early biochips were based on the concept of a DNA microarray, which is a piece of glass, plastic or silicon substrate on which pieces of DNA, i.e., probes, have been affixed. There are a number of commercial microarrays available in the marketplace today, e.g., GeneChip DNAarray from Affymetrix, NanoChip microarray from Nanogen, and DNA microarray from Agilent. A drawback of these arrays is that they are "passive chips"; they are neither reconfigurable nor can they be used for sample preparation. Product cost is also a problem; for example, while the microarray chips from Affymetrix cost in the range of $\$ 500-\$ 2000$ each, the accompanying instrument and licensing/royalty fees can be as high as $\$ 175000$.

The basic idea of a microfluidic biochip is to integrate all necessary functions for biochemical analysis using microfluidics technology. These micro-total-analysis-systems are more versatile than microarrays. Integrated functions include assay operations, detection, and sample preparation.

\section{A. Continuous-Flow Microfluidics}

Traditional (continuous-flow) microfluidic technologies are based on the continuous flow of liquid through micro-fabricated channels [8], [10]-[16]. Continuous-flow systems are inherently difficult to integrate because the parameters that govern flow field (e.g. pressure, fluid resistance, electric field strength) vary along the flow-path, making the flow at any location dependent upon the properties of the entire system. Moreover, unavoidable shear flow and diffusion in microchannels make it difficult to eliminate intersample contamination and dead volumes. Furthermore, since structure and functionality are so tightly coupled, each system is only appropriate for a narrow class of applications.

\section{B. Digital Microfluidics}

A digital microfluidic biochip utilizes electrowetting on dielectric (EWOD) to manipulate and move microliter or nanoliter droplets containing biological samples on a two-dimensional electrode array [2]-[4], [17], [23]-[26]. A unit cell in the array includes a pair of electrodes that acts as two parallel plates. The bottom plate contains a patterned array of individually controlled electrodes, and the top plate is coated with a continuous ground electrode. A droplet rests on a hydrophobic surface over an electrode, as shown in Fig. 1. It is moved by applying a control voltage to an electrode adjacent to the droplet and, at the same time, deactivating the electrode just under the droplet. Using interfacial tension gradients, droplets can be moved to any location on a two-dimensional array. A film of silicone oil is used as a filler medium to prevent cross contamination and evaporation [7], [27]. In Fig. 1(a), the electrode pitch is $1.5 \mathrm{~mm}$ and the gap height is $600 \mu \mathrm{m}$. Recent work has demonstrated chips with an electrode pitch of $60 \mu \mathrm{m}$ and $19.2 \mu \mathrm{m}$ height. To

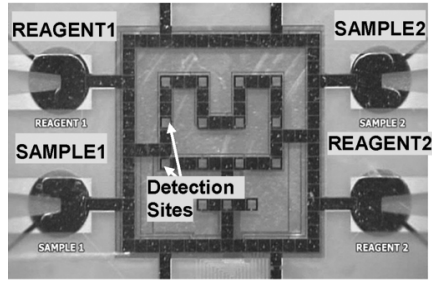

(a)

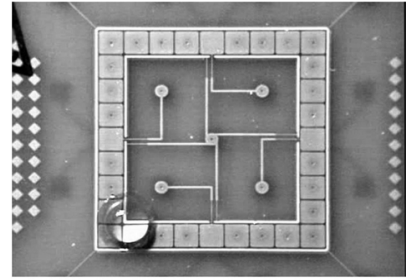

(b)
Fig. 1. Fabricated digital microfluidic arrays. (a) Glass substrate [25]. (b) PCB substrate [57].

dispense a $105 \mathrm{pl}$ droplet, an actuation voltage of $80 \mathrm{~V}$ is required [74].

An alternative method for digital microfluidics, namely dielectrophoresis (DEP), relies on AC actuation [28]-[31]. However, excessive Joule heating is often seen as a problem for DEP. In contrast to DEP actuation, Joule heating is virtually eliminated in EWOD because the dielectric layer covering the electrodes blocks DC electric current.

The division of a volume of fluid into discrete, independently controllable "packets" or droplets, provides several advantages over continuous-flow. The reduction of microfluidics to a set of basic repeated operations (i.e., "move one unit of fluid one distance unit") allows a hierarchical and cell-based design approach to be utilized. By varying the patterns of control-voltage activation (a clock signal with logic-high and logic-low values), many fluid-handling operations such as droplet merging, splitting, mixing, and dispensing can be easily executed. The digital microfluidic platform offers dynamic reconfigurability, since fluidic operations can be performed anywhere on the array. Droplet routes and operation scheduling results are programmed into a microcontroller that drives electrodes in the array. As a result, there is no need for dedicated on-chip reaction chambers. Reservoirs are included on the array boundary, from which droplets can be easily dispensed [4]. The disposable nature of these chips precludes multiple uses over long periods of time; nevertheless, reconfigurability allows the same chip design and fabrication method to be used for multiple applications. As in the case of today's integrated circuits, such multifunctional chips facilitate mass production and lower product cost.

To address the need for low-cost, printed circuit board (PCB) technology has been employed for inexpensive fabrication [32]. Using a copper layer for the electrodes, solder mask as the insulator, and a Teflon AF coating for hydrophobicity, the microfluidic array can be fabricated using an existing PCB process. A typical coplanar digital microfluidic chip has an electrode pitch of $1.5 \mathrm{~mm}$, with a gap of $90 \mu \mathrm{m}$ [73]. Actuation voltages of around $220 \mathrm{~V}$ are applied for fluidic operation. Power supplies are, therefore, external to the microfluidic chip.

Demonstrated applications of digital microfluidics include the on-chip detection of explosives such as commercial-grade 2,4,6-trinitrotoluene (TNT) and pure 2,4-dinitrotoluene [6], automated on-chip measurement of airborne particulate matter [21], [22], and colorimetric assays [7]. Measured performance metrics for such colorimetric assays have been reported in prior work, e.g., [2], [7]. Digital microfluidic biochips are being designed for on-chip gene sequencing through synthesis [4], 
and integrated hematology, pathology, molecular diagnostics, cytology, microbiology, and serology on the same platform [18]. A prototype has been developed for pyrosequencing [4], which targets the simultaneous execution of 106 fluidic operations and the processing of billions of droplets. Other lab-on-chip/biochip systems are being designed for protein crystallization, which requires the concurrent execution of hundreds of operations [33], [34]. A commercially available droplet-based (using dielectrophoresis) lab-on-chip embeds more than $600,00020 \mu \mathrm{m}$ by $20 \mu \mathrm{m}$ electrodes with integrated optical detectors [35]. Experimental work using digital microfluidics has been focused on in vitro and ex vivo techniques, as opposed to in vivo methods.

A limitation of digital microfluidics is the nonspecific adsorption of reagents and samples to the electrode surface. Therefore, design techniques must be developed to avoid cross-contamination. Another limitation today is the difficulty in ensuring accuracy and reproducibility for the droplet split operation. Finally, the world-to-chip interface is a challenge since it is difficult to deliver reagents and samples to such biochips. Nevertheless, these issues are being studied in ongoing research, e.g., [72], [74], and there is considerable enthusiasm for this emerging technology. Many droplet operations, e.g., droplet dispensing and mixing, have been demonstrated to be repeatable with high accuracy [27].

In a recent review paper on the use of microfluidics for protein crystallization [36], the following question was posed: can we purchase identical crystallization devices, produced under adequate quality control? The authors go on to say, "Drawing upon integrated circuits as an analogy, microfluidics devices may be reducible to a standard set of discrete operations which can then be custom assembled to form more complex operations as needed. With this approach, the success of manufacturing investment does not have to rest upon a single application."

The discrete droplet-based biochip described in this paper is perfectly suited as a platform technology, since it avoids the common pitfall of custom devices offered by other continuous-flow microfluidic technologies.

\section{SYNTHESIS METHODS}

In this section, we examine a progression of CAD problems related to biochip synthesis.

\section{A. Scheduling and Module Placement}

Recent years have seen growing interest in the automated design and synthesis of microfluidic biochips [39], [44], [47]-[51], [53]-[56], [59]-[61], [63]-[67], [69]. Optimization goals here include the minimization of assay completion time, minimization of chip area, and higher defect tolerance. The minimization of the assay completion time, i.e., the maximization of throughput, is essential for environmental monitoring applications where sensors can provide early warning. Real-time response is also necessary for surgery and neonatal clinical diagnostics. Finally, biological samples are sensitive to the environment and to temperature variations, and it is difficult to maintain an optimal clinical or laboratory environment on chip. To ensure the integrity of assay results, it is therefore desirable to minimize the time that samples spend on-chip before assay results are obtained.

Increased throughput also improves operational reliability. Long assay durations imply that high actuation voltages need to be maintained on some electrodes, which accelerate insulator degradation and dielectric breakdown, reducing the number of assays that can be performed on a chip during its lifetime.

One of the first published methods for biochip synthesis decoupled high-level synthesis from physical design [44], [54]. Architectural-level synthesis for microfluidic biochips can be viewed as the problem of scheduling assay functions and binding them to a given number of resources so as to maximize parallelism, thereby decreasing response time. A behavioral model for a set of bioassays is first obtained from their laboratory protocols. Architectural-level synthesis is then used to generate a macroscopic structure of the biochip; this is analogous to a structural register-transfer level (RTL) model in electronic CAD [40]. On the other hand, geometry-level synthesis (physical design) addresses the placement of resources and the routing of droplets to satisfy objectives such as area or throughput. It creates the final layout of the biochip, consisting of the placement of microfluidic modules such as mixers and storage units, the routes that droplets take between different modules, and other geometrical details [53].

As in the case of high-level synthesis for integrated circuits, resource binding in the biochip synthesis flow refers to the mapping from bioassay operations to available functional resources. Note that there may be several types of resources for any given bioassay operation. For example, a $2 \times 2$ array mixer, a $2 \times 3$ array mixer and a $2 \times 4$ array mixer can be used for a droplet mixing operation, but with different mixing times. In such cases, a resource selection procedure must be used. On the other hand, resource binding may associate one functional resource with several assay operations; this necessitates resource sharing. Once resource binding is carried out, the time duration for each bioassay operation can be easily determined. Scheduling determines the start times and stop times of all assay operations, subject to the precedence and resource-sharing constraints.

A key problem in the geometry-level synthesis of biochips is the placement of microfluidic modules such as different types of mixers and storage units. Since digital microfluidics-based biochips enable dynamic reconfiguration of the microfluidic array during run-time, they allow the placement of different modules on the same location during different time intervals. A simulated annealing-based heuristic approach has been developed to solve the NP-complete problem in a computationally efficient manner [53]. Solutions for the placement problem can provide the designer with guidelines on the size of the array to be manufactured. If module placement is carried out for a fabricated array, area minimization frees up more unit cells for sample collection and preparation.

Architectural synthesis is based on rough estimates for placement costs such as the area of the microfluidic modules. These estimates provide lower bounds on the exact biochip area, since the overheads due to spare cells and cells used for droplet transportation are not known a priori. However, it cannot be accurately predicted if the biochip design meets system specifications, e.g., maximum allowable array area and upper limits 


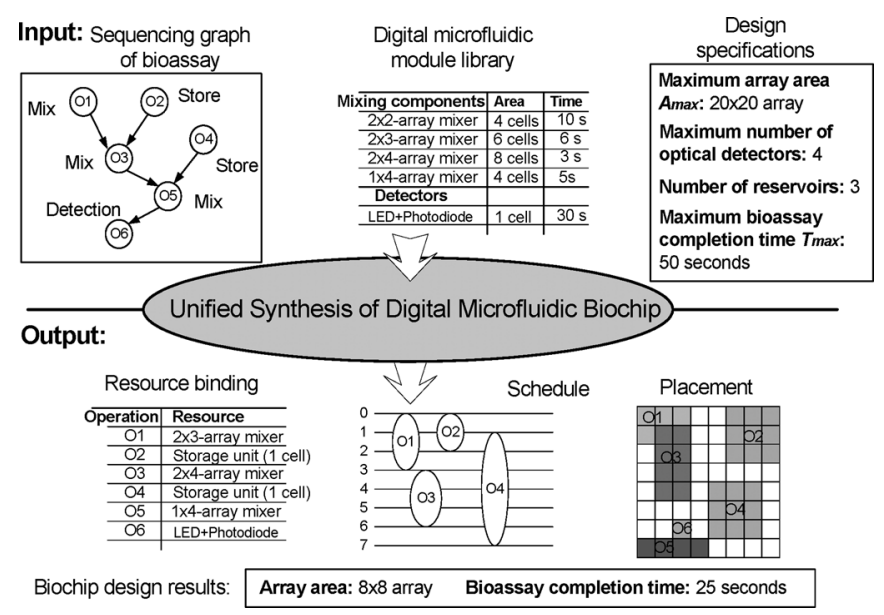

Fig. 2. An example illustrating system-level synthesis [47].

on assay completion times, until both high-level synthesis and physical design are carried out. [47] proposed a unified systemlevel synthesis method for microfluidic biochips based on parallel recombinative simulated annealing (PRSA), which offers a link between these two steps. This method allows users to describe bioassays at a high level of abstraction, and it automatically maps behavioral descriptions to the underlying microfluidic array.

The design flow is illustrated in Fig. 2. First, the different bioassay operations (e.g. mixing and dilution), and their mutual dependences are represented using a sequencing graph. Next, a combination of simulated annealing and genetic algorithms are used for unified resource binding, operation scheduling, and module placement. A chromosome is used to represent each candidate solution, i.e., a design point. The word chromosome is derived from genetics, and it encodes the characteristics of an individual in the population. In each chromosome, operations are randomly bound to resources. Based on the binding results, list scheduling is used to determine the start times of operations, i.e., each operation starts with a random latency after its scheduled time. Finally, a module placement is derived based on the resource binding and the schedule of fluidic operations. A weighted sum of area- and time-cost is used to evaluate the quality of the design. The design is improved through a series of genetic evolutions based on PRSA. It generates an optimized schedule of bioassay operations, the binding of assay operations to resources, and a layout of the microfluidic biochip.

Efficient reconfiguration techniques have been developed to bypass faulty unit cells in the microfluidic array [55]. A microfluidic module containing a faulty unit cell can easily be relocated to another part of the microfluidic array by changing the control voltages applied to the corresponding electrodes [49]. Defect tolerance can also be achieved by including redundant elements in the system; these elements can be used to replace faulty elements through reconfiguration techniques [48]. Another method is based on graceful degradation, in which all elements in the system are treated in a uniform manner, and no element is designated as a spare [50]. In the presence of defects, a subsystem with no faulty element is first determined from the faulty system. This subsystem provides the desired functionality, but with a gracefully degraded level of performance (e.g., longer execution times). Due to the dynamic reconfigurability of digital microfluidics-based biochips, microfluidic components (e.g., mixers) can be viewed as reconfigurable virtual devices. For example, a $2 \times 4$ array mixer (implemented using a rectangular array of control electrodes-two in the $\mathrm{X}$-direction and four in Y-direction) can easily be reconfigured to a $2 \times 3$ array mixer or a $2 \times 2$ array mixer.

Fig. 3(a) shows the module placement results and the microfluidic array design for a representative protein assay [47]. The XY-plane refers to the placement of modules on the chip real estate. The Z-axis refers to time. As shown in Fig. 3(b), we can further integrate optical detectors as well as on-chip reservoirs/dispensing ports into the microfluidic array to form a complete digital microfluidic biochip for the protein assay. Fig. 3(c) shows the corresponding results when some of the unit cells in the array are faulty, and reconfiguration is used in a unified manner with synthesis. The solution obtained for the fault-free array yields a biochip design with a $9 \times 9$ microfluidic array and the completion time for the protein assay is $363 \mathrm{~s}$. The design for the faulty array allows the protein assay to operate with an increase of only $6 \%$ in the completion time, i.e., the completion time is now 385 seconds.

Note that there are clear similarities between the programmability of dynamically reconfigurable field-programmable gate-arrays (DR-FPGAs) and digital microfluidics. However, there are also some key differences. The programmability of DR-FPGAs is limited by the well-defined roles of interconnect and logic blocks. Interconnect cannot be used for storing information, and logic blocks cannot be used for routing. In contrast, digital microfluidics-based biochips offer significantly more programmability. The cells in the microfluidic array can be used for storage and functional operations, as well as for transporting fluid droplets. Moreover, "virtual devices" in digital microfluidics can be easily moved without incurring overhead. Non-reconfigurable devices such as reservoirs and detectors also have to be considered.

The top-down synthesis flow described above unifies architecture level design with physical-level module placement. However, it suffers from two drawbacks. For operation scheduling, it is assumed that the time cost for droplet routing is negligible, which implies that droplet routing has no influence on the operation completion time. While generating physical layouts, the synthesis tool in [47] provides only the layouts of the modules and it leaves droplet routing pathways unspecified. The assumption of negligible droplet transportation times is valid for small microfluidic arrays. However, for large arrays and for biochemical protocols that require several concurrent fluidic operations on-chip, the droplet transportation time is significant and routing complexity is non-trivial. This problem is addressed in the next subsection.

\section{B. Droplet Routing}

A key problem in biochip physical design is droplet routing between modules, and between modules and I/O ports (i.e., on-chip reservoirs). The dynamic reconfigurability inherent in digital microfluidics allows different droplet routes to share cells on the microfluidic array during different time intervals. In this sense, the routes in microfluidic biochips can be viewed as virtual routes, which make droplet routing different from 


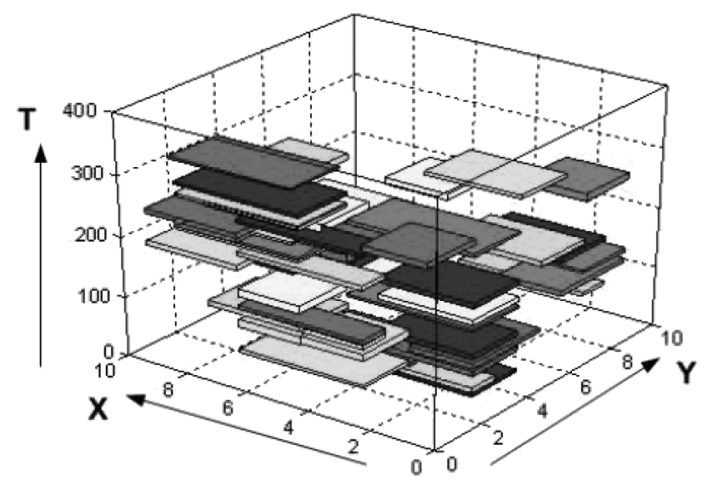

(a)

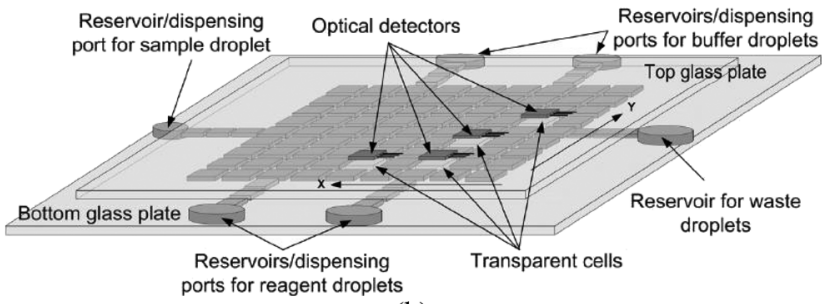

(b)

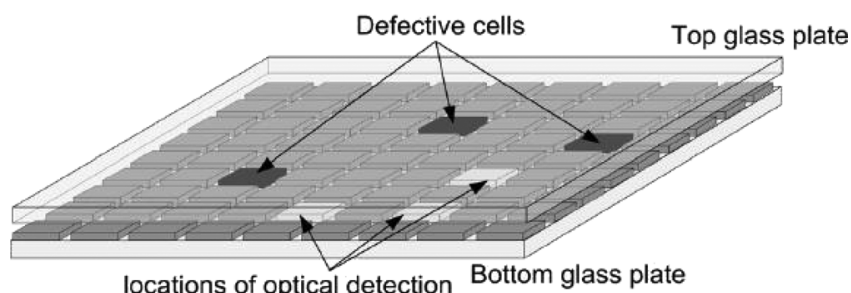

locations of optical detection Bottom glass plate

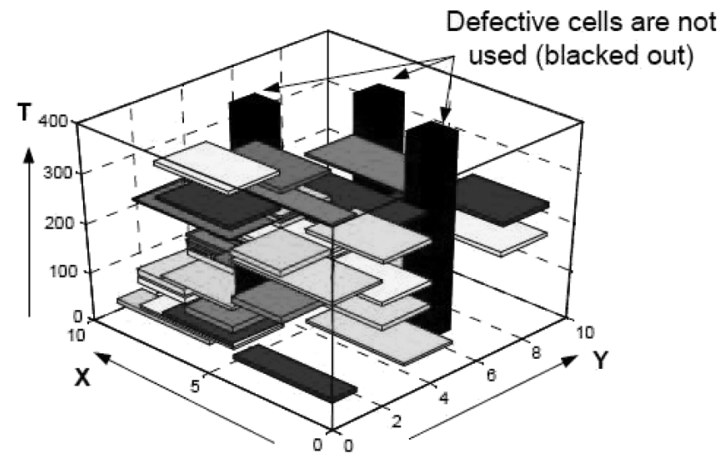

(c)

Fig. 3. (a) A 3-D model illustrating the synthesis results. (b) A digital microfluidic biochip for a protein assay. (c) A defective array and module placement for the protein assay on this array.

the classical wire VLSI routing problem. Systematic routing method for digital microfluidic biochips have therefore been developed to minimize the number of cells used for droplet routing, while satisfying constraints imposed by performance goals and fluidic properties.

One of the first methods for droplet routing in biochips was published in [51]. The main objective in routing is to find droplet routes with minimum lengths, where route length is measured by the number of cells in the path from the starting point to the destination. For a microfluidic array of fixed size, minimum-length droplet routes lead to the minimization of the total number of cells used in droplet routing, thus freeing up more spare cells for fault tolerance. As in the case of electronic circuits, the fluidic ports on the boundary of microfluidic mod- ules are referred to as pins. Similarly, we refer to the droplet routes between pins of different modules or on-chip reservoirs as nets. Thus, a fluidic route on which a single droplet is transported between two terminals can easily be modeled as a 2-pin net. We also need to move two droplets from different terminals to one common microfluidic module (e.g., mixer) for mixing. To allow droplet mixing simultaneously during their transport, we need to model such fluidic routes using 3-pin nets.

During droplet routing, a minimum spacing between droplets must be maintained to prevent accidental mixing, except for the case when droplet merging is desired (e.g., in 3-pin nets). Fluidic constraint rules in [51] need to be satisfied in order to avoid undesirable mixing. We view the microfluidic modules placed on the array as obstacles in droplet routing. In order to avoid conflicts between droplet routes and assay operations, a segregation region is added to wrap around the functional region of microfluidic modules. Another constraint in droplet routing is given by an upper limit on droplet transportation time. The delay for each droplet route should not exceed some maximum, e.g., $10 \%$ of a time-slot used in scheduling, in order that the droplet-routing time can be ignored for scheduling assay operations [51].

Since a digital microfluidic array can be reconfigured dynamically at run-time, a series of 2-D placement configurations of modules in different time spans are obtained in the module placement phase [48]. Therefore, the droplet routing is decomposed into a series of sub-problems. We obtain a complete droplet-routing solution by solving these sub-problems sequentially.

Based on this problem formulation, a two-stage routing method has been proposed in [51]. In the first stage, $M$ alternative routes for each net are generated. In the second stage, a single route from the $M$ alternatives for each net is selected independent of the routing order of nets. This method also exploits the features of dynamic reconfigurability and independent controllability of electrodes to modify droplet pathways to override potential violation of fluidic constraints.

Droplet routing should be considered in the synthesis flow for digital microfluidics, in order to generate a routable synthesized design for the availability of routing paths. [59] proposed a method to incorporate droplet-routability in the PRSA-based synthesis flow. This method estimates the droplet-routability using two metrics. It adopts the average module distance (over all interdependent modules) as the first design metric to guarantee the routability of modules in the synthesized biochip. It also adopts the maximum module distance as the second design metric to approximate the maximum length of droplet manipulation. Since synthesis results with high routability values are more likely to lead to simple and efficient droplet pathways, this method incorporates the above two metrics into the fitness function by a factor that can be fine-tuned according to different design specifications to control the PRSA-based procedure. For each chromosome considered in the PRSA-based synthesis flow, this method calculates both the average and maximum module distance. Candidate designs with low routability are discarded during evolution. Thus, the synthesis procedure guarantees that the routing complexity is reduced for the synthesized biochip, while meeting constraints on array size and bioassay processing time. 


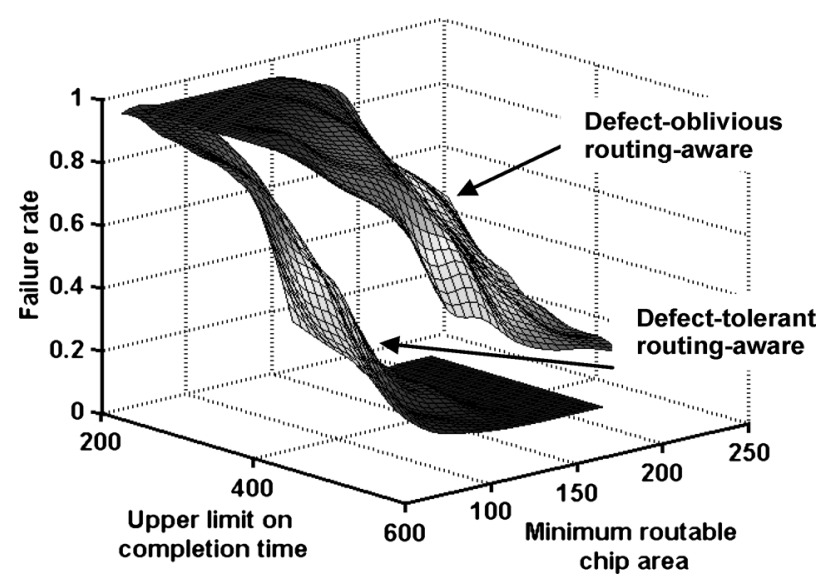

Fig. 4. Feasibility frontier surface and feasible design region for defect-tolerant and defect-oblivious routing-aware synthesis methods.

We ran the defect-tolerant routing-aware and defect-oblivious routing-aware algorithms under a set of combinations of weights in the fitness function for the protein assay example. We carried out random defect injection into each design and obtain its failure rate. We mapped each design $\mathrm{G}$ to a three-dimensional point $\left(T_{G}, A_{G}, F_{G}\right)$, where $T_{G}, A_{G}, F_{G}$ are completion time, chip area, and failure rate of the design, respectively. A point $\left(T_{G}, A_{G}, F_{G}\right)$ is referred to as a feasibility boundary point if there are no other points $\left(T_{m}, A_{m}, F_{m}\right)$ such that $T_{m}<T_{G}$, $A_{m}<A_{G}$, and $F_{m}<F_{G}$. A feasibility frontier surface is obtained by connecting all the feasibility boundary points, as shown in Fig. 4. The feasible design region corresponds to the space above the feasible surface. Any design specification can be met whose corresponding is point located in this region; otherwise, no feasible design exists for this specification. As shown in Fig. 4, defect-tolerant routing-aware synthesis leads to a lower-feasibility frontier surface and a larger feasible design space as compared to the defect-oblivious method.

Cross-contamination is likely to occur when multiple droplet routes intersect or overlap with each other. At the intersection site of two droplet routes, a droplet that arrives at a later clock cycle can be contaminated by the residue left behind by another droplet that passed through at an earlier clock cycle. Disjoint droplet routes are utilized to avoid the cross-contamination. In a set of disjoint routes, a droplet route does not share any cell in its path with each of the other droplet routes in that set. Such routes eliminate the possibility of a droplet being transported via a cell when another droplet has already passed through it in an earlier time interval. The problem of finding feasible disjoint routes for 2-pin or 3-pin nets in the 2-D microfluidic array can be directly mapped to the problem of finding disjoint paths (vertex-disjoint or edge-disjoint) for pairs of vertices in a graph, which has been shown to be NP-complete [42].

Reference [69] proposed a droplet-routing method that avoids cross-contamination in the optimization of droplet flow paths. The proposed approach targets disjoint droplet routes and minimizes the number of cells used for droplet routing. A net-routing ordering method is used to obtain an optimized order for the routing of all the nets in a sub-problem. Each net in the net-routing order is routed sequentially. The cells occupied by the routed paths are marked as obstacles for the

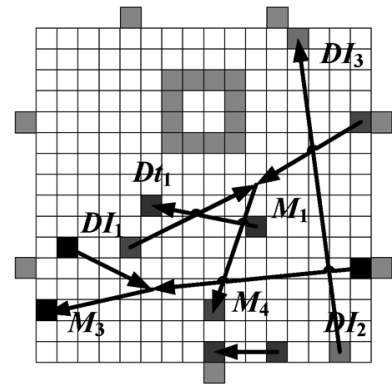

(a)

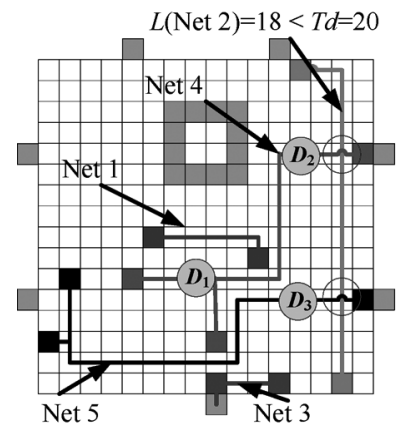

(c)

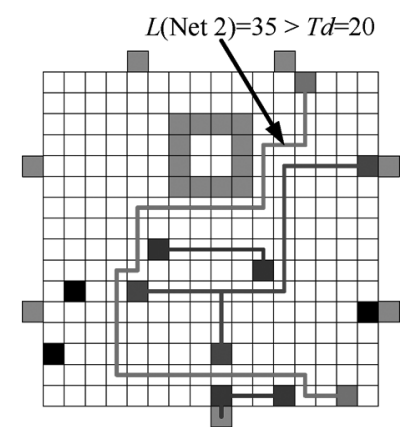

(b)

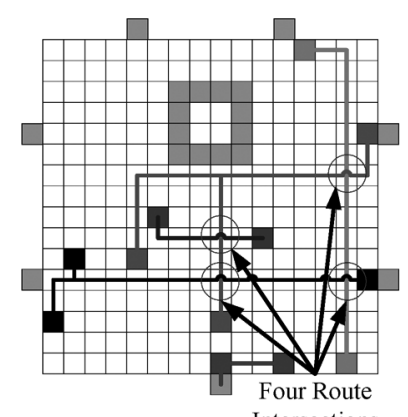

(d)
Fig. 5. Disjoint routing and cross-contamination-oblivious routing for a subproblem. (a) Three 2-pin nets and two 3-pin nets. (b) Net 2 violates the timing delay constraint. (c) feasible routes for all the nets using the disjoint-routing method. (d) routing results obtained using the cross-contamination-oblivious routing method.

unrouted nets. Therefore, the latter route is vertex-disjoint with respect to all the previous routes. Since it is often difficult to find vertex-disjoint paths to solve the droplet-routing problem, it is more practical to relax the overlap restriction and search for edge-disjoint routes in such cases.

Fig. 5 illustrates the application of the proposed routing method to a sub-problem. As shown in Fig. 5(a), there are three 2-pin nets and two 3-pin nets. The net-routing order is Net 3, Net 1, Net 4, Net 2, and Net 5. While generating the route for Net 2, since the paths for previous routed Net 3, Net 1 and Net 4 have been marked as obstacles, the shortest paths between $\mathrm{DI}_{2}$ and $D I_{3}$ (i.e., Net 2) violate the timing delay constraint, as shown in Fig. 5(b). Thus, we put Net 2 to the conflict list, then generate the route for Net 5 . After the route for Net 5 is generated, we generate the route for Net 2 without considering previously generated paths as obstacles. A route that has no common pair of adjacent cells with the previously routed paths is selected. The obtained route for Net 2 satisfies the timing constraint, as shown in Fig. 5(c). The route for Net 2 induces two intersections (cross-contamination sites) with the routes for Net 4 and Net 5, respectively. Therefore, the desirable edge-disjoint droplet-route set with total 63 cells is finally obtained. We compare the routing results obtained using the proposed method for this sub-problem with a baseline method that utilizes the modified Lee algorithm in [51] without considering cross-contamination between different routes. The baseline method uses four route intersections (cross-contamination sites), two more than that obtained by the proposed method, as shown in Fig. 5(d). 
Since cross-contamination can also happen across two subproblems, after the droplet-routing process in one sub-problem, a wash operation needs to be introduced. In a wash operation, a wash droplet is routed to traverse selected cells and remove residue from them. However, performing wash operations after every sub-problem significantly increases the total time needed for droplet routing for all the sub-problems. Therefore, [69] proposed an optimization model to minimize the number of wash operations that must be used between successive routing steps.

\section{Pin-Constrained ChIP DESIGN}

Electrode addressing is an important problem in biochip design. It refers to the manner in which electrodes are connected to and controlled by input pins. Early design-automation techniques relied on the availability of a direct-addressing scheme. For large arrays, direct-addressing schemes lead to a large number of control pins, and the associated interconnect routing problem significantly adds to the product cost. Thus, the design of pin-constrained digital microfluidic arrays is of great practical importance for the emerging marketplace. In this section, we describe a number of pin-constrained biochip design methods.

\section{A. Droplet-Trace-Based Array Partitioning}

An array-partitioning-based pin-constrained design method of digital microfluidic biochips proposed in [56]. This method uses array partitioning and careful pin assignment to reduce the number of control pins. The key idea is to "virtually" partition the array into regions. The partitioning criterion here is to ensure at most one droplet is included in each partition. The droplet trace, defined as the set of cells traversed by a single droplet, serves as the basis for generating the array partitions. The droplet trace can be easily extracted from the droplet routing information and the placement of the modules to which it is routed. If droplets traces intersect on the array, the partitions derived by this method overlap in some regions. Sets of pins from an "overlapping" partition cannot be used in the overlapped region since the reuse of the pins may lead to droplet interference. The solution to this problem is to make the overlapping region a new partition, referred to as the overlapping partition, and use direct addressing (one-to-one mapping) for it.

A Connect-5 algorithm is used to address the problem of how to map control pins to the electrodes in a partition, which can be easily implemented using a 3-layer-PCB. The Connect-5 algorithm succeeds in avoiding droplet interference while moving a single droplet inside the partition. It can be integrated into the droplet-trace-based array partitioning method to generate droplet-interference-free layouts with a minimum number of pins. However, this method requires detailed information about the scheduling of assay operations, microfluidic module placement, and droplet routing pathways. Thus, the array design in such cases is specific to a target biofluidic application.

\section{B. Cross-Referencing-Based Droplet Manipulation}

An alternative method based on a cross-reference driving scheme is presented in [60]. This method allows control of an $N \times M$ grid array with only $N+M$ control pins. The electrode rows are patterned on both the top and bottom plates, and placed orthogonally. In order to drive a droplet along the $\mathrm{X}$-direction, electrode rows on the bottom plate serve as driving electrodes, while electrode rows on the top serve as reference ground electrodes. The roles are reversed for movement along the Y-direction. This cross-reference method facilitates the reduction of control pins. However, due to electrode interference, this design cannot handle the simultaneous movement of more than two droplets. For the concurrent manipulation of multiple droplets on a cross-referencing-based biochip, multiple row and column pins must be selected to activate the destination cells, i.e., cells to which the droplets are supposed to move. However, the selected row and column pins may also result in the activation of cells other than the intended droplet destinations.

A solution based on destination-cell categorization is proposed to tackle the above problem. The key idea is to group the droplet movements according to their destination cells. A group consists of droplets whose destination cells share the same column or row. In this way, the manipulation of multiple droplets is ordered in time; droplets in the same group can be moved simultaneously without electrode interference, but the movements for the different groups must be sequential. The problem of finding the minimum number of groups can be directly mapped to the problem of determining a minimal clique partition from graph theory [37]. A linear-time heuristic algorithm based on row-scanning and column-scanning has been used to derive the clique partitions.

\section{Broadcast-Addressing Method}

One drawback of the cross-reference driving scheme is that this design requires a special electrode structure (i.e., both top and bottom plates contain electrode rows), which results in increased manufacturing cost. Thereby, a broadcast-addressing based design technique for pin-constrained and multi-functional biochips has been developed in [61].

To execute a specific bioassay, routing and scheduling information must be stored in the form of electrode activation sequences, where each bit representing the status of the electrode at a specific time-step. The status can be either " 1 " (activate), "0" (deactivate) or "F" (floating). The "floating" status is represented using the symbol " $\mathrm{x}$ " and refer to it as "don't-care". Each electrode activation sequence contains several don't-care terms, which can be replaced by " 1 " or " 0 ". If two sequences can be made identical by careful replacing these don't-care terms with " 0 " or " 1 ", they are referred to as compatible sequences. Compatible sequences can be generated from a single signal source.

The number of control pins can be reduced by connecting together electrodes with mutually-compatible activation sequences, and addressing them using a single control pin. Therefore, the resulting electrode-access method is referred to as a broadcast addressing. The first step here is to partition the electrodes into groups. For all the electrodes in any group, the corresponding activation sequences must be pairwise-compatible. The problem of finding an optimal partition that leads to the minimum number of groups can be easily mapped to the problem of determining a minimal clique partition from graph theory [37]. The minimum number of groups yields the minimum number of control pins. 
TABLE I

EXAMPLES of Fault Models for Digital Microfluidic Biochip [57]

\begin{tabular}{|c|c|c|c|c|}
\hline Cause of defect & Defect type & $\begin{array}{l}\text { Number } \\
\text { of cells }\end{array}$ & Fault model & Observable error \\
\hline $\begin{array}{l}\text { Excessive actuation voltage } \\
\text { applied to an electrode }\end{array}$ & Dielectric breakdown & 1 & $\begin{array}{l}\text { Droplet-electrode short (a } \\
\text { short between the droplet and } \\
\text { the electrode) }\end{array}$ & $\begin{array}{l}\text { Droplet undergoes electrolysis, which } \\
\text { prevents its further transportation }\end{array}$ \\
\hline $\begin{array}{l}\text { Electrode actuation for } \\
\text { excessive duration }\end{array}$ & $\begin{array}{l}\text { Irreversible charge concentration } \\
\text { on an electrode }\end{array}$ & 1 & $\begin{array}{l}\text { Electrode-stuck-on (the } \\
\text { electrode remains constantly } \\
\text { activated) }\end{array}$ & $\begin{array}{l}\text { Unintentional droplet operations or } \\
\text { stuck droplets }\end{array}$ \\
\hline $\begin{array}{l}\text { Excessive mechanical force } \\
\text { applied to the chip }\end{array}$ & $\begin{array}{l}\text { Misalignment of parallel plates } \\
\text { (electrodes and ground plane) }\end{array}$ & 1 & $\begin{array}{l}\text { Pressure gradient (net static } \\
\text { pressure in some direction) }\end{array}$ & $\begin{array}{l}\text { Droplet transportation without } \\
\text { activation voltage }\end{array}$ \\
\hline Coating failure & Non-uniform dielectric layer & 1 & $\begin{array}{l}\text { Dielectric islands (islands of } \\
\text { Teflon coating) }\end{array}$ & $\begin{array}{l}\text { Fragmentation of droplets and their } \\
\text { motion is prevented }\end{array}$ \\
\hline \multirow{3}{*}{$\begin{array}{l}\text { Abnormal metal layer } \\
\text { deposition and etch variation } \\
\text { during fabrication }\end{array}$} & Grounding Failure & 1 & $\begin{array}{l}\text { Floating droplets (droplet are } \\
\text { not anchored) }\end{array}$ & Failure of droplet transportation \\
\hline & Broken wire to control source & 1 & $\begin{array}{l}\text { Electrode open (electrode } \\
\text { actuation is not possible) }\end{array}$ & $\begin{array}{l}\text { Failure to activate the electrode for } \\
\text { droplet transportation }\end{array}$ \\
\hline & $\begin{array}{l}\text { Metal connection between two } \\
\text { adjacent electrodes }\end{array}$ & 2 & $\begin{array}{l}\text { Electrode short (short between } \\
\text { electrodes) }\end{array}$ & \multirow{2}{*}{$\begin{array}{l}\text { A droplet resides in the middle of the } \\
\text { two shorted electrodes, and its transport } \\
\text { along one or more directions cannot be } \\
\text { achieved }\end{array}$} \\
\hline $\begin{array}{l}\text { Particle contamination or liquid } \\
\text { residue }\end{array}$ & $\begin{array}{l}\text { A particle that connect two } \\
\text { adjacent electrodes }\end{array}$ & 2 & Electrode short & \\
\hline \multirow{2}{*}{$\begin{array}{l}\text { Protein adsorption during } \\
\text { bioassay [17] }\end{array}$} & \multirow{2}{*}{$\begin{array}{l}\text { Sample residue on electrode } \\
\text { surface }\end{array}$} & \multirow[t]{2}{*}{1} & Resistive open at electrode & Droplet transportation is impeded. \\
\hline & & & Contamination & $\begin{array}{l}\text { Assay results are outside the range of } \\
\text { possible outcomes }\end{array}$ \\
\hline
\end{tabular}

\section{TESTING AND DiAgnosis}

In this section, we describe recent advances in the testing of digital microfluidic biochips and fault localization techniques.

\section{A. Fault Modeling}

As in microelectronic circuits, a defective microfluidic biochip is said to have a failure if its operation does not match its specified behavior [41]. In order to facilitate the detection of defects, fault models that efficiently represent the effect of physical defects at some level of abstraction are required. These models can be used to capture the effect of physical defects that produce incorrect behaviors in the electrical or fluidic domain. As described in [43], faults in digital microfluidic systems can be classified as being either catastrophic or parametric. Catastrophic faults lead to a complete malfunction of the system, while parametric faults cause degradation in the system performance. A parametric fault is detectable only if this deviation exceeds the tolerance in system performance.

Catastrophic may be caused by a number of physical defects, for example:

- Dielectric breakdown: The breakdown of the dielectric at high voltage levels creates a short between the droplet and the electrode. When this happens, the droplet undergoes electrolysis, thereby preventing further transportation.

- Short between the adjacent electrodes: If a short occurs between two adjacent electrodes, the two electrodes effectively form one longer electrode. When a droplet resides on this electrode, it is no longer large enough to overlap the gap between adjacent electrodes. As a result, the actuation of the droplet can no longer be achieved.

- Degradation of the insulator: This degradation effect is unpredictable and may become apparent gradually during the operation of the microfluidic system. A consequence is that droplets often fragment and their motion is prevented because of the unwanted variation of surface tension forces along their flow path.
- Open in the metal connection between the electrode and the control source: This defect results in a failure in activating the electrode for transport.

Table I lists some common failure sources, defects and the corresponding fault models for catastrophic faults in digital microfluidic lab-on-chip. Examples of some common parametric faults include the following:

- Geometrical parameter deviation: The deviation in insulator thickness, electrode length and height between parallel plates may exceed their tolerance value.

- Change in viscosity of droplet and filler medium. These can occur during operation due to an unexpected biochemical reaction, or changes in operational environment, e.g., temperature variation.

\section{B. Structural Test Techniques}

A unified test methodology for digital microfluidic biochips has recently been presented, whereby faults can be detected by controlling and tracking droplet motion electrically [45]. Test stimuli droplets containing a conductive fluid (e.g., KCL solution) are dispensed from the droplet source. These droplets are guided through the unit cells following the test plan towards the droplet sink, which is connected to an integrated capacitive detection circuit. Details of the capacitive readout circuit are presented in [58]. Most catastrophic faults result in a complete cessation of droplet transportation. Therefore, we can determine the fault-free or faulty status of the system by simply observing the arrival of test stimuli droplets at selected ports. An efficient test plan ensures that testing does not conflict with the normal bioassay, and it guides test stimuli droplets to cover all the unit cells available for testing. The microfluidic array can be modeled as an undirected graph, and the pathway for the test droplet can be determined by solving the Hamiltonian path problem 
[52]. With negligible hardware overhead, this method also offers an opportunity to implement self-test for microfluidic systems and therefore eliminate the need for costly, bulky, and expensive external test equipment. Furthermore, after detection, droplet flow paths for bioassays can be reconfigured dynamically such that faulty unit cells are bypassed without interrupting the normal operation.

Even though most catastrophic faults lead to a complete cessation of droplet transportation, there exist differences between their corresponding erroneous behaviors. For instance, to test for the electrode-open fault, it is sufficient to move a test droplet from any adjacent cell to the faulty cell. The droplet will always be stuck during its motion due to the failure in charging the control electrode. On the other hand, if we move a test droplet across the faulty cells affected by an electrode-short fault, the test droplet may or may not be stuck depending on its flow direction. Therefore, to detect such faults, it is not enough to solve only the Hamiltonian path problem. In [46], a solution based on Euler paths in graphs is described for detecting electrode shorts.

Despite its effectiveness for detecting electrode shorts, testing based on an Euler path suffers from long test application time. This approach uses only one droplet to traverse the microfluidic array, irrespective of the array size. Fault diagnosis is carried out by using multiple test application steps and adaptive Euler paths. Such a diagnosis method is inefficient since defect-free cells are tested multiple times. Moreover, the test method leads to a test plan that is specific to a target biochip. If the array dimensions are changed, the test plan must be completely altered. In addition, to facilitate chip testing in the field, test plans need to be programmed into a microcontroller. However, the hardware implementations of test plans from [45] are expensive, especially for low cost, disposable biochips. More recently, a cost-effective testing methodology referred to as "parallel scan-like test" has been proposed [58]. The method is named thus because it manipulates multiple test droplets in parallel to traverse the target microfluidic array, just as test stimuli can be applied in parallel to the different scan chains in an integrated circuit.

The parallel scan-like test method has been applied to a fabricated biochip. The chip-under-test is a PCB microfluidic platform for DNA sequencing, as shown in Fig. 6. The platform consists of a $7 \times 7$ array, 8 reservoirs and routing electrodes that connect reservoirs to the array. A total of 9 cells are reserved for grounding, and they are not available for droplet transportation. As a baseline, Euler-path-based testing was applied to this chip [58]. The test procedure takes $57 \mathrm{~s}$, assuming a (typical) $1-\mathrm{Hz}$ electrode-actuation frequency. Next, parallel scan-like test was applied to this chip. The test application procedure takes $46 \mathrm{sec}-$ onds, again for a $1-\mathrm{Hz}$ actuation frequency.

Next we evaluate the time needed for fault diagnosis for the two methods. In [58], a fabricated chip was used, which was known a priori to contain one defect. For the Euler-path-based method, binary search was carried out to locate the defective cell. A total of seven iterations were needed and the total diagnosis time was $173 \mathrm{~s}$. On the other hand, parallel scan-like test can simply determine the defect site from test-outcome readouts. No additional diagnosis steps are needed and the diagnosis time is the same as the testing time, i.e., $44 \mathrm{~s}$, which correspond to a $75 \%$ reduction compared to [45].

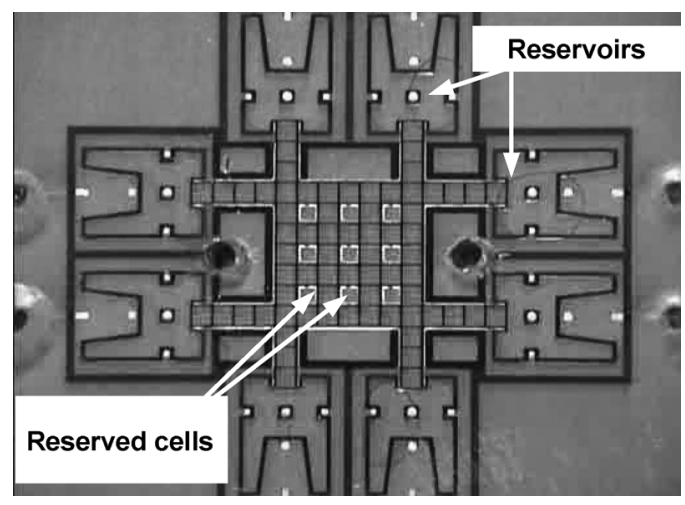

Fig. 6. Fabricated lab-on-chip for DNA sequencing used to demonstrate parallel scan-like testing.

A drawback of the above "structural" test methods is that they focus only on physical defects, and they overlook module functionality. Therefore, these methods can only guarantee that a biochip is defect-free. However, a defect-free microfluidic array can also malfunction in many ways. For example, a defect-free reservoir may result in large volume variations when droplets are dispensed from it. A splitter composed of three defect-free electrodes may split a big droplet into two droplets with significantly unbalanced volumes. These phenomena, referred to as malfunctions, are not the result of electrode defects. Instead, they are activated only for certain patterns of droplet movement or fluidic operations. Such malfunctions can have serious consequences on the integrity of bioassay results.

\section{Functional Testing Techniques}

Functional testing involves test procedures to check whether groups of cells can be used to perform certain operations, e.g., droplet mixing and splitting. For the test of a specific operation, the corresponding patterns of droplet movement are carried out on the target cluster of cells. If a target cell cluster fails the test, e.g., the mixing test, we label it as a malfunctioning cluster. As in the case of structural testing, fault models must be developed for functional testing. Malfunctions in fluidic operations are identified and included in the list of faults; see Table II.

Functional test methods to detect the defects and malfunctions have recently been developed. In particular, dispensing test, mixing test, splitting test, and capacitive sensing test have been described in [57] to address the corresponding malfunctions.

Functional test methods were applied to a PCB microfluidic platform for the Polymerase Chain Reaction (PCR), as shown in Fig. 7. The platform consists of two columns and two rows of electrodes, three reservoirs, and routing electrodes that connect the reservoirs to the array. A dispensing malfunction is shown in Fig. 8.

An illustration of the mixing and splitting test is shown in Fig. 9. The bottom row was first targeted and five test droplets were dispensed to the odd electrodes, as shown in Fig. 9(a). Next, splitting test for the even electrodes was carried out. Droplets were split and merged on the even electrodes. In Fig. 9(b), we see a series of droplets of the same volume resting on the even electrodes, which means that all the odd electrodes 
TABLE II

FunCTIONAL FAULT MODELS [57]

\begin{tabular}{|l|l|c|l|l|}
\hline Cause of malfunction & Malfunction type & $\begin{array}{l}\text { Number } \\
\text { of cells }\end{array}$ & Fault model & Observable error \\
\hline $\begin{array}{l}\text { Electrode actuation for } \\
\text { excessive duration }\end{array}$ & $\begin{array}{l}\text { Irreversible charge } \\
\text { concentration on the dispensing } \\
\text { electrode }\end{array}$ & 3 & $\begin{array}{l}\text { Dispensing-stuck-on } \\
\text { (droplet is dispensed by not } \\
\text { fully cut off from the } \\
\text { reservoir) }\end{array}$ & $\begin{array}{l}\text { No droplet can be dispensed from the } \\
\text { reservoir }\end{array}$ \\
\hline $\begin{array}{l}\text { Electrode shape variation in } \\
\text { fabrication }\end{array}$ & Deformity of electrodes & 3 & $\begin{array}{l}\text { No overlap between droplets } \\
\text { to be mixed and center } \\
\text { electrode }\end{array}$ & Mixing failure \\
\hline $\begin{array}{l}\text { Electrode electrostatic } \\
\text { property variation in } \\
\text { fabrication }\end{array}$ & Unequal actuation voltages & 3 & $\begin{array}{l}\text { Pressure gradient (net static } \\
\text { pressure in some direction) }\end{array}$ & Unbalanced volumes of split droplets \\
\hline Bad soldering & $\begin{array}{l}\text { Parasitic capacitance in the } \\
\text { capacitive sensing circuit }\end{array}$ & 1 & $\begin{array}{l}\text { Oversensitive or insensitive } \\
\text { capacitive sensing }\end{array}$ & False positive/negative in detection \\
\hline
\end{tabular}

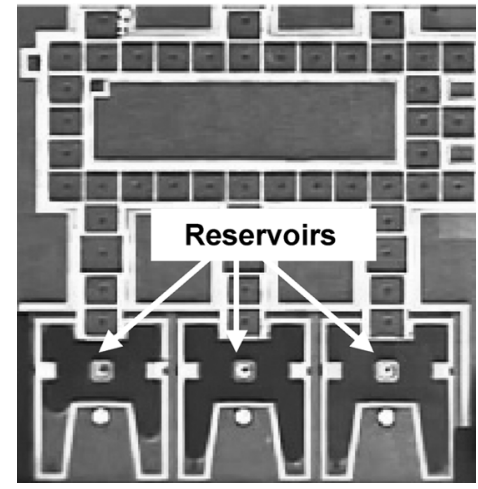

Fig. 7. Fabricated lab-on-chip used for PCR.

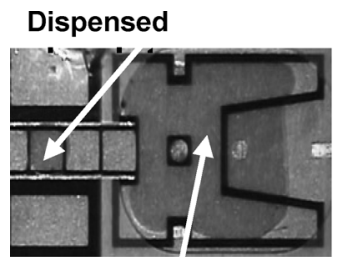

Reservoir

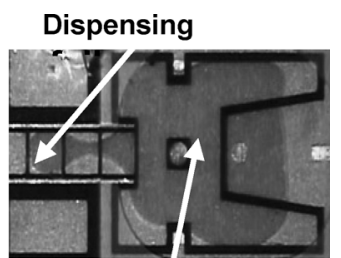

Reservoir
Fig. 8. Illustration of (a) normal dispensing and (b) dispensing failure, for a fabricated lab-on-chip.

passed the splitting test, and merging at the even electrodes worked well. However, when the splitting test was carried out on the even electrodes, a large variation in droplet volume was observed on the 3rd and 5th electrodes; see Fig. 9(c). This variation implied a malfunction, leading to unbalanced splitting on the 4th electrode. The malfunction was detected when the droplets were routed to the capacitive sensing circuit. The 4th electrode on the bottom row was marked as an unqualified splitting site.

\section{Built-In Self-Test (BIST) Techniques}

Previous test methods for digital microfluidic platforms use capacitive sensing circuits to read and analyze test outcomes. After reading the test-outcome droplets in a consecutive manner, the capacitive sensing circuit generates a pulse-sequence corresponding to the detection of these droplets. This approach requires an additional step to analyze the pulse sequence to determine whether the microfluidic array under-test is defective. The

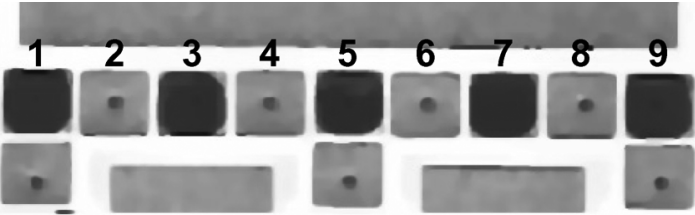

(a)

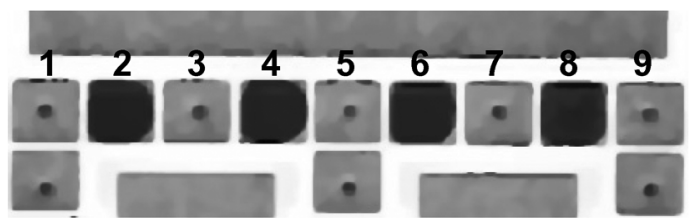

(b)

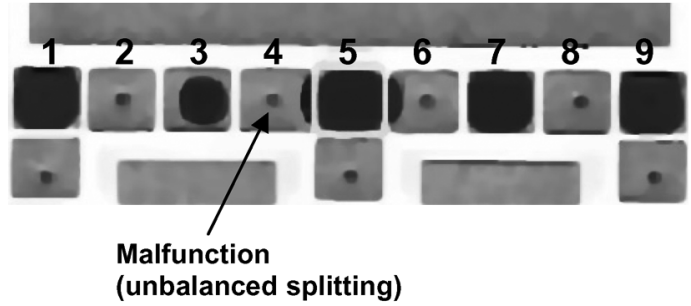

(c)

Fig. 9. Mxing and splitting test for a fabricated PCR chip.

reading of test outcomes and the analysis of pulse sequences increase test time; the latter procedure is especially prone to errors arising from inaccuracies in sensor calibration. The complexity of the capacitive-sensing circuit and the need for pulse-sequence analysis make previously proposed testing methods less practical, especially for field operation.

A built-in self-test (BIST) method for digital microfluidic lab-on-chip is proposed in [68]. This method utilizes microfluidic logic gates to implement the "compactor" in a BIST architecture. Using the principle of electrowetting-ondielectric, microfluidic AND, OR and NOT gates are implemented through basic droplet-handling operations such as transportation, merging, and splitting.

The definitions of logic values ' 0 ' or ' 1 ' are as follows: the presence of a droplet of $1 \times$ volume at an input or output port indicates a logic value of ' 1 '. The absence of a droplet at an input or output port indicates the logic value ' 0 '. The same interpretations at inputs and outputs enable the output of one gate to be 


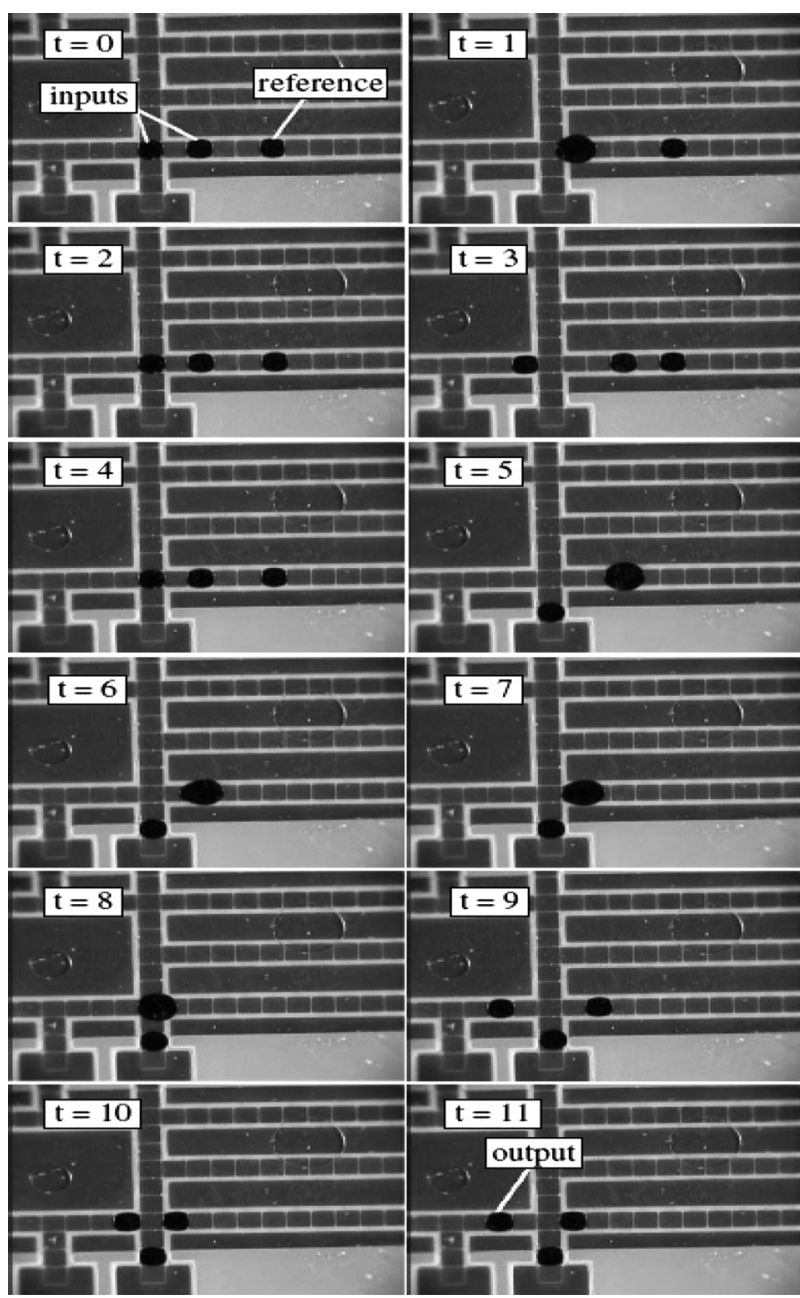

Fig. 10. On-chip cycle-by-cycle operation for the microfluidic OR gate with input 11 .

fed as an input to another gate, thus logic gates can be easily cascaded.

Fig. 10 shows the operation of the OR gate for two inputs $X_{1} X_{2}=11$. For this chip, the electrode pitch was $1.5 \mathrm{~mm}$ and the gap was $0.475 \mathrm{~mm}$. The voltage used for splitting was $250 \mathrm{~V}$. The voltage used for transportation was $80 \mathrm{~V}$. The clock frequency was $1 \mathrm{~Hz}$.

When $t=0$, two $1 \times$ droplets stay on the electrodes representing two inputs, while one $1 \times$ droplet stays on the electrode representing the reference port. When $t=1$, two $1 \times$ droplets from the input ports merge together to form a $2 \times$ droplet. From $t=2$ to 4 , the $2 \times$ droplet splits into two $1 \times$ droplets. Note that the splitting step in the experiment occupies five electrodes and lasts for three clock cycles. When $t=5$, one $1 \times$ droplet mixes with the $1 \times$ droplet from the reference port to form a $2 \times$ droplet, while the other $1 \times$ droplet moves downward to the waste reservoir. From $t=6$ to 8 , the merged $2 \times$ droplet moves to the crossing electrode. From $t=9$ to 10 , the $2 \times$ droplet splits into two $1 \times$ droplets. At $t=11$, there is one $1 \times$ droplet on the electrode representing the output, which indicates that the output value of microfluidic OR gate with inputs $X_{1} X_{2}=11$ is 1 .

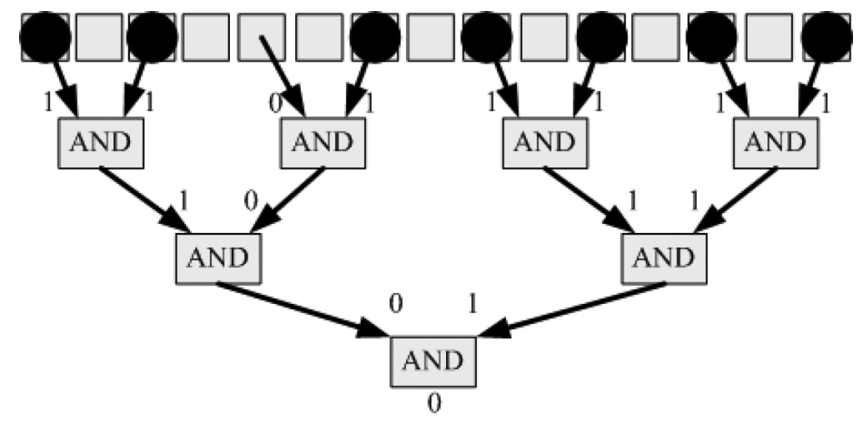

Fig. 11. Schematic of a microfluidic compactor for parallel scan-like testing: compactor output $={ }^{\prime} 0$ '.

The microfluidic compactor can compress the test-outcome droplets of both structural test and functional test into one droplet in a very short amount of time, and the droplet can be detected using a simple photodiode detector, thereby avoiding the need for a capacitive-sensing circuit and complicated pulse-sequence analysis. An efficient diagnosis method based on a "microfluidic encoder" is also proposed to locate a single defective electrode in a microfluidic array. Fig. 11 illustrates the schematic of the microfluidic compactor for parallel scan-like test of odd rows/columns in a $16 \times 16$ microfluidic array. The electrodes represent the last row/column where the pseudosinks are located. The compactor consists of three layers of microfluidic 2-input AND gates. The output of the AND gate in the third layer is connected to the photo-diode detector located in the sink reservoir of the microfluidic array. The arrows show the direction of droplet routing through the AND gates. The number at each arrow indicates the logic value of the input or output, according to the definitions for logic values in microfluidic systems. In Fig. 11, after the parallel scan-like test for odd rows/columns, there is no droplet on the fifth electrode, indicating that the corresponding row/column has a defect. Therefore, the left input of the second AND gate in the first layer is ' 0 ', while other input values are ' 1 '. The output of the AND gate with a ' 0 ' input value is ' 0 '. No droplet appears on the output port (electrode) of the microfluidic compactor, therefore the output value of the compactor is ' 0 '. Instead of routing all the droplets in odd pseudo-sinks one-by-one to the capacitive-sensing circuit and analyzing the resulting pulse-sequence, we use the photo-diode detector to check for the presence/absence of the $1 \times$ droplet on the output of the compactor. Since no droplet is detected by the photo-diode detector, we conclude that there is a defect in the odd rows/columns under test.

\section{E. Design for Testability}

Previous pin-constrained design methods achieve a significant reduction in the number of input pins needed for controlling the electrodes. However, as a trade-off, droplet manipulation steps must satisfy additional constraints. These constraints can result in test procedures being either completely ineffective or effective only for a small part of the chip. As a result, chip testability is significantly reduced. Note that the reduction in testability is due to the conflicts between the fluidic operation steps required by functional test and the constraints on droplet manipulations introduced by the mapping of pins to electrodes. Fig. 12 shows a pin-constrained chip design for a representative 


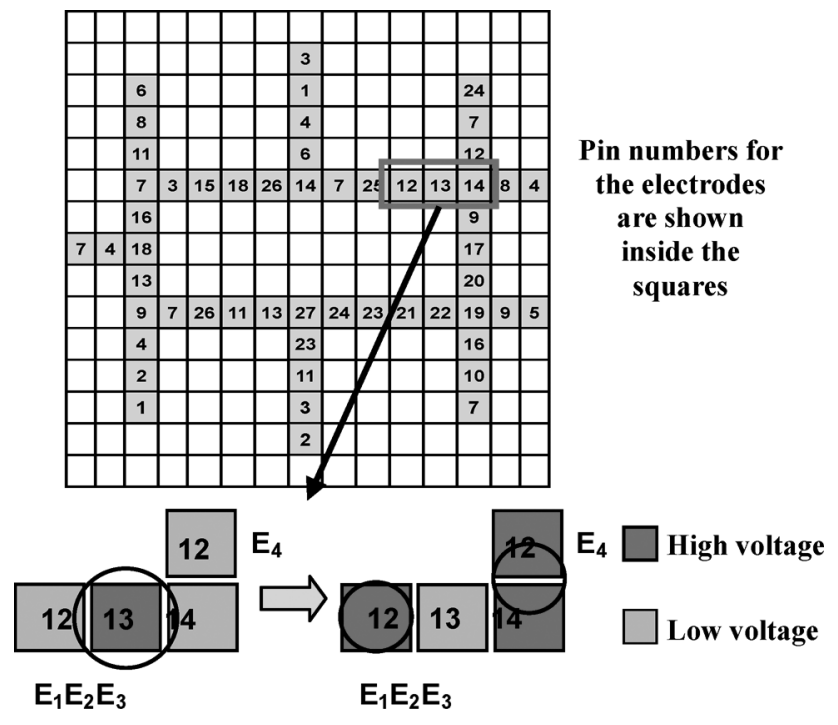

Fig. 12. An example of an untestable functional unit on a pin-constrained chip for multiplexed assay.

protein-dilution assay. The functional test procedure requires a splitting operation to be executed on the highlighted functional unit. To do this, we first activate Pin 13 to hold a test droplet at $\mathrm{E}_{2}$. Next, we deactivate Pin 13 and activate Pin 12 and Pin 14 to split the test droplet into two small droplets seated on $\mathrm{E}_{1}$ and $\mathrm{E}_{3}$. However, $\mathrm{E}_{4}$ is also charged by activating Pin 12 . As a result, the split droplet that is supposed to be seated on $\mathrm{E}_{2}$ will be moved unintentionally to the boundary of $\mathrm{E}_{4}$ and $\mathrm{E}_{3}$. Note that different mappings for a pin-constrained chip lead to different untestable functional units, thereby different levels of chip testability.

The authors in [62] proposed a design-for-testability (DFT) solution to facilitate the testing in pin-constrained design. A testaware pin-constrained design method that incorporates test procedures into the fluidic manipulation steps in the target bioassay protocol is proposed. First, the fluidic operations required by the test procedure are derived from the scheduling and routing steps related to the test droplets. Next, we merge these fluidic operations with the droplet manipulation steps needed for the target bioassay. The merging can be carried out by attaching the electrode-activation sequences for the test procedure to the electrode-activation sequences for the target bioassays. For each electrode in the array, its activation sequence during the test procedure is added to that for the target bioassay to form a longer sequence. These merged longer electrode-activation sequences are provided as input to the broadcast-addressing method, thereby the resulted chip design will support not only the target bioassay but also the test operations. Fig. 13 shows a linear array consisting of four electrodes. A simple "routing assay" is mapped to the array, where a droplet is to be routed from E4 to E1, one electrode per step. We first list the activation sequence for each electrode (Table T1) in Fig. 13. Next we add a splitting test on E3. The electrode-activation sequences for the splitting test are shown in Table T2 of Fig. 13. These activation sequences are then combined with the activation sequences in $\mathrm{T} 1$. The resulted

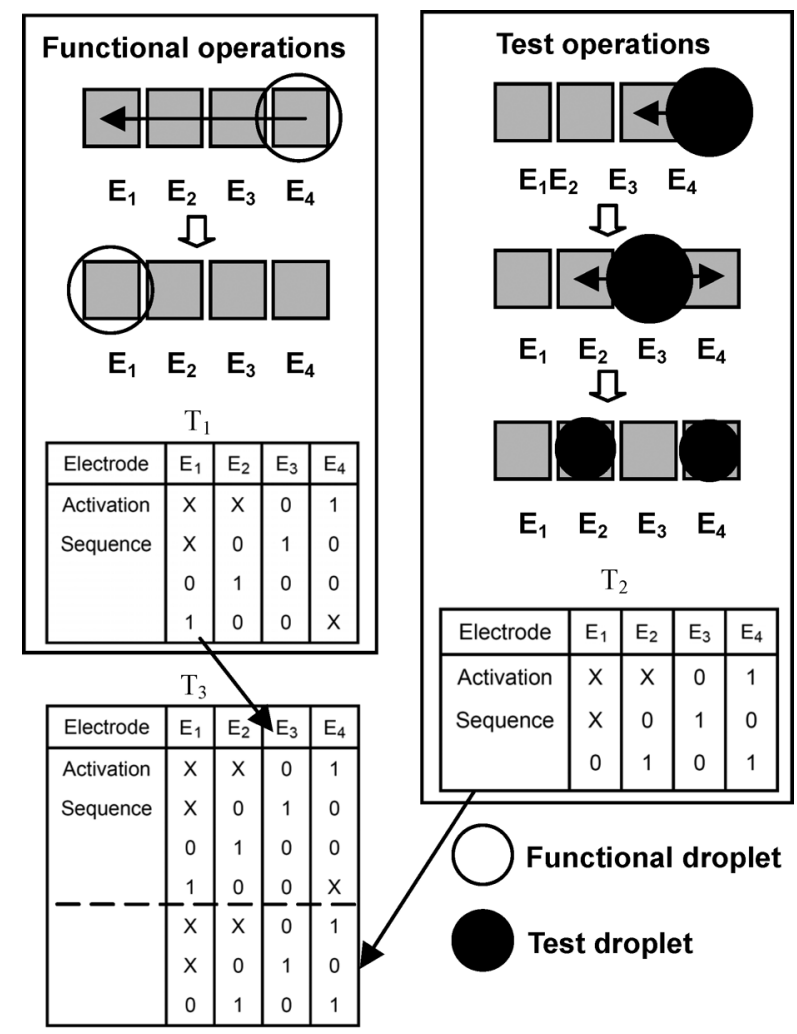

Fig. 13. Illustration of the influence by adding test operations to the bioassay.

longer activation sequences are listed in table T3. The broadcast-addressing method is then applied to generate the eventual pin assignment according to sequences in $\mathrm{T} 3$.

By applying pin-constrained design to the testability-aware bioassay protocol, the proposed method ensures that the resulting chip layout supports the effective execution of test-related droplet operations for the entire chip. Therefore, the proposed DFT method allows design of pin-constrained biochips with a high level of testability with negligible overhead in terms of the number of control pins.

\section{CONCLUSION}

We have presented a survey of research on design automation and test techniques for digital microfluidic biochips. We first provided an overview of the digital microfluidic platform, and then highlighted advances in synthesis and droplet routing techniques. Practical design techniques for achieving high throughout with a small number of control pins have been presented. Testing and design-for-testability techniques have also been presented. Common defects have been identified and related to logical fault models. Based on these fault models, test techniques for emerging lab-on-chip devices and digital microfluidic modules have been presented. The use of these test techniques for fabricated devices has been highlighted. These design techniques are expected to pave the way for the deployment and use of biochips in the emerging marketplace. As the next step for research in this field, there is a need to integrate 
more on-chip sensors such that adaptive decision making can be carried out on-chip for biomolecular recognition.

\section{ACKNOWLEDGMENT}

The author thanks Y. Zhao and Z. Zhang of Duke University, Durham, NC, for help in the preparation of this manuscript. The author also thanks a number of colleagues at Duke University and at Advanced Liquid Logic, Inc., who have contributed to this work. They include Dr. F. Su, Dr. T. Xu, Y. Zhao, W. Hwang, Dr. P. Paik, Dr. V. Pamula, and Prof. R. B. Fair.

\section{REFERENCES}

[1] T. H. Schulte, R. L. Bardell, and B. H. Weigl, "Microfluidic technologies in clinical diagnostics," Clinica Chim. Acta, vol. 321, pp. 1-10, 2002.

[2] V. Srinvasan, V. K. Pamula, M. G. Pollack, and R. B. Fair, "Clinical diagnostics on human whole blood, plasma, serum, urine, saliva, sweat, and tears on a digital microfluidic platform," in Proc. MicroTAS, 2003, pp. 1287-1290.

[3] A. Guiseppi-Elie, S. Brahim, G. Slaughter, and K. R. Ward, "Design of a subcutaneous implantable biochip for monitoring of glucose and lactate," IEEE Sensors J., vol. 5, pp. 345-355, 2005.

[4] R. B. Fair, A. Khlystov, T. D. Tailor, V. Ivanov, R. D. Evans, P. B. Griffin, V. Srinivasan, V. K. Pamula, M. G. Pollack, and J. Zhou, "Chemical and biological applications of digital-microfluidic devices," IEEE Design \& Test of Comput., vol. 24, pp. 10-24, 2007.

[5] E. A. Ottesen, J. W. Hong, S. R. Quake, and J. R. Leadbetter, "Microfluidic digital PCR enables multigene analysis of individual environmental bacteria," Science, vol. 314, pp. 1464-1467, 2006.

[6] Y. Zhao and S. K. Cho, "Microparticle sampling by electrowetting actuated droplet sweeping," Lab on a Chip, vol. 6, pp. 137-144, 2006.

[7] V. Srinivasan, V. K. Pamula, and R. B. Fair, "An integrated digital microfluidic lab-on-a-chip for clinical diagnostics on human physiological fluids," Lab on a Chip, vol. 4, pp. 310-315, 2004.

[8] E. Verpoorte and N. F. D. Rooij, "Microfluidics meets MEMS," Proceedings of IEEE, vol. 91, pp. 930-953, 2003.

[9] Semiconductor Industry Association, International Technology Roadmap for Semiconductors (ITRS) 2007 [Online]. Available: http://www.itrs.net/Links/2007ITRS/Home2007.htm

[10] R. Qiao and N. R. Aluru, "A compact model for electroosmotic flows in microfluidic devices," J. Micromechanics and Microengineering, vol. 12, pp. 625-635, 2002.

[11] Y. Wang, Q. Lin, and T. Mukherjee, "A model for laminar diffusionbased complex electrokinetic passive micromixers," Lab on a Chip, vol. 5, pp. 877-887, 2005

[12] A. S. Bedekar, Y. Wang, S. Krishnamoorthy, S. S. Siddhaye, and S. Sundaram, "System-level simulation of flow-induced dispersion in lab-on-a-chip systems," IEEE Trans. Comput.-Aided Des. Integr. Circuits Syst., vol. 25, pp. 294-304, 2006.

[13] Y. Wang, Q. Lin, and T. Mukherjee, "Composable behavioral models and schematic-based simulation of electrokinetic lab-on-a-chip systems," IEEE Trans. Comput.-Aided Des. Integr. Circuits Syst., vol. 25, pp. 258-273, 2006.

[14] X. Chen, D. F. Cui, C. Liu, H. Li, and J. Chen, "Continuous flow microfluidic device for cell separation, cell lysis and DNA purification," Anal. Chim. Acta, vol. 584, pp. 237-243, 2007.

[15] CoventorWareTM [Online]. Available: http://www.coventor.com/ coventorware.html

[16] T. Korsmeyer, J. Zeng, and K. Greiner, "Design tools for BioMEMS," in Proc.IEEE/ACM Design Autom. Conf., 2004, pp. 622-627.

[17] M. G. Pollack, R. B. Fair, and A. D. Shenderov, "Electrowetting-based actuation of liquid droplets for microfluidic applications," Appl. Phys. Lett., vol. 77, pp. 1725-1726, 2000.

[18] R. B. Fair, V. Srinivasan, H. Ren, P. Paik, V. K. Pamula, and M. G. Pollack, "Electrowetting-based on-chip sample processing for integrated microfluidics," in Proc. IEEE Int. Electron Devices Meeting (IEDM), 2003, pp. 32.5.1-32.5.4.

[19] S. K. Cho, H. J. Moon, and C. J. Kim, "Creating, transporting, cutting, and merging liquid droplets by electrowetting-based actuation for digital microfluidic circuits," J. Microelectromech. Syst., vol. 12, pp. 70-80, Feb. 2003.
[20] A. R. Wheeler, H. Moon, C. A. Bird, R. R. O. Loo, C.-J. Kim, J. A. Loo, and R. L. Garrell, "Digital microfluidics with in-line sample purification for proteomics analyses with MALDI-MS," Anal. Chem., vol. 77, pp. 534-540, 2005.

[21] J. Gong and C. J. Kim, "Two-dimensional digital microfluidic system by multi-layer printed circuit board," in Proc, IEEE MEMS, 2005, pp. 726-729.

[22] D. Chatterjee, B. Hetayothin, A. R. Wheeler, D. J. King, and R. L. Garrell, "Droplet-based microfluidics with nonaqueous solvents and solutions," Lab on a Chip, vol. 6, pp. 199-206, 2006.

[23] J. Berthier, Microdrops and Digital Microfluidics: Processing, Development, and Applications (Micro \& Nano Technologies). : William Andrew, 2007.

[24] H. Ren, R. B. Fair, and M. G. Pollack, "Automated on-chip droplet dispensing with volume control by electro-wetting actuation and capacitance metering," Sensors and Actuators B-Chemical, vol. 98, pp. 319-327, 2004.

[25] V. Srinivasan, V. K. Pamula, and R. B. Fair, "Droplet-based microfluidic lab-on-a-chip for glucose detection," Anal. Chim. Acta, vol. 507, pp. 145-150, 2004.

[26] Advanced Liquid Logic [Online]. Available: http://www.liquidlogic.com

[27] P. Y. Paik, "Rapid Droplet Mixers for Digital Microfluidic Systems," M. S. Thesis, Duke Univ., Durham, NC, 2003.

[28] T. B. Jones, M. Gunji, M. Washizu, and M. J. Feldman, "Dielectrophoretic liquid actuation and nanodroplet formation," J. Appl. Phys., vol. 89, pp. 1441-1448, 2001.

[29] J. Vykoukal, J. Schwartz, F. Becker, and P. Gascoyne, "A programmable dielectric fluid processor for droplet-based chemistry," in Proc. Micro Total Analysis Syst., 2001, pp. 72-74.

[30] T. B. Jones, K. L. Wang, and D. J. Yao, "Frequency-dependent electromechanics of aqueous liquids: Electrowetting and dielectrophoresis," Langmuir, vol. 20, pp. 2813-2818, 2004.

[31] J. Gong and C. J. Kim, "Two-dimensional digital microfluidic system by multi-layer printed circuit board," in Proc. IEEE MEMS, 2005, pp. 726-729.

[32] [Online]. Available: http://www.ultimatepcb.com/index.php

[33] T. Xu, P. Thwar, V. Srinivasan, V. K. Pamula, and K. Chakrabarty, "Digital microfluidic biochip for protein crystallization," in IEEE-NIH Life Science Syst. and Appl. Workshop, Bethesda, MD, 2007.

[34] T. Xu, K. Chakrabarty, and V. K. Pamula, "Design and optimization of a digital microfluidic biochip for protein crystallization," in Proc. IEEE/ACM Int. Conf. on Computer-Aided Design, 2008, pp. 297-301.

[35] Silicon Biosystems [Online]. Available: www.siliconbiosystems.com

[36] M. Van Der Woerd, D. Ferree, and M. Pusey, "The promise of macromolecular crystallization in microfluidic chips," J. Struct. Biol., vol. 142, pp. 180-187, 2003.

[37] R. Diestel, Graph Theory. Berlin, Germany: Springer, 2005.

[38] X. Chen, D. F. Cui, C. Liu, H. Li, and J. Chen, "Continuous flow microfluidic device for cell separation, cell lysis and DNA purification," Anal. Chim. Acta, vol. 584, pp. 237-243, 2007.

[39] E. Maftei, P. Pop, J. Madsen, and T. Stidsen, "Placement-aware architectural synthesis of digital microfluidic biochips using ILP," in Proc. Int. Conf. on Very Large Scale Integr. of System on Chip, 2008, pp. $425-430$

[40] G. De Micheli, Synthesis and Optimization of Digital Circuits. New York: McGraw-Hill, 1994.

[41] H. G. Kerkhoff, "Testing of microelectronic-biofluidic systems," IEEE Design \& Test of Computers, vol. 24, pp. 72-82, 2007.

[42] M. E. Kramer and J. van Leeuwen, "The complexity of wire routing and finding the minimum area layouts for arbitrary VLSI circuits," in Adv. Comput. Res. 2: VLSI Theory. London, U.K.: JAI Press, 1984.

[43] F. Su, S. Ozev, and K. Chakrabarty, "Testing of droplet-based microelectrofluidic systems," in Proc. IEEE Int. Test Conf., 2003, pp. $1192-1200$.

[44] F. Su and K. Chakrabarty, "Architectural-level synthesis of digital microfluidics-based biochips," in Proc. IEEE Int. Conf. on CAD, 2004, pp. 223-228.

[45] F. Su, S. Ozev, and K. Chakrabarty, "Ensuring the operational health of droplet-based microelectrofluidic biosensor systems," IEEE Sensors J., vol. 5, pp. 763-773, 2005.

[46] F. Su, W. Hwang, A. Mukherjee, and K. Chakrabarty, "Testing and diagnosis of realistic defects in digital microfluidic biochips," J. Electronic Testing: Theory and Appl., vol. 23, pp. 219-233, 2007.

[47] F. Su and K. Chakrabarty, "Unified high-level synthesis and module placement for defect-tolerant microfluidic biochips," in Proc. IEEE/ACM Design Autom. Conf., 2005, pp. 825-830. 
[48] F. Su and K. Chakrabarty, "Design of fault-tolerant and dynamicallyreconfigurable microfluidic biochips," in Proc. DATE Conf., 2005, pp. 1202-1207.

[49] F. Su and K. Chakrabarty, "Reconfiguration techniques for digital microfluidic biochips," in Proc. IEEE Design, Test, Integr. and Packaging of MEMS/MOEMS Symposium, 2005, pp. 143-148.

[50] F. Su and K. Chakrabarty, "Defect tolerance for gracefully-degradable microfluidics-based biochips," in Proc. IEEE VLSI Test Symp., 2005, pp. 321-326.

[51] F. Su, W. Hwang, and K. Chakrabarty, "Droplet routing in the synthesis of digital microfluidic biochips," in Proc. Design, Autom. and Test in Eur. (DATE) Conf., 2006, pp. 323-328.

[52] F. Su, S. Ozev, and K. Chakrabarty, "Test planning and test resource optimization for droplet-based microfluidic systems," J. Electronic Testing: Theory and Applications, vol. 22, pp. 199-210, 2006.

[53] F. Su and K. Chakrabarty, "Module placement for fault-tolerant microfluidics-based biochips," ACM Trans. Design Autom. Electron. Syst., vol. 11, pp. 682-710, 2006.

[54] F. Su and K. Chakrabarty, "High-level synthesis of digital microfluidic biochips," ACM J. Emerging Technol. in Comput. Syst., vol. 3, 2008, Article 16

[55] F. Su, "Synthesis, Testing, and Reconfiguration Techniques for Digital Microfluidic Biochips," Ph.D. dissertation, Duke Univ., Durham, NC, USA, 2006.

[56] T. Xu and K. Chakrabarty, "Droplet-trace-based array partitioning and a pin assignment algorithm for the automated design of digital microfluidic biochips," in Proc. IEEE/ACM Int. Conf. on Hardware/Software Codesign and System Synthesis, 2006, pp. 112-117.

[57] T. Xu and K. Chakrabarty, "Functional testing of digital microfluidic biochips," in Proc. IEEE Int. Test Conf., 2007.

[58] T. Xu and K. Chakrabarty, "Parallel scan-like test and multiple-defect diagnosis for digital microfluidic biochips," IEEE Trans. Biomed. Circuits Syst., vol. 1, no. 2, pp. 148-158, Jun.e 2007.

[59] T. Xu and K. Chakrabarty, "Integrated droplet routing in the synthesis of microfluidic biochips," in Proc. IEEE/ACM Design Automation Conf., 2007, pp. 948-953.

[60] T. Xu and K. Chakrabarty, "A cross-referencing-based droplet manipulation method for high-throughput and pin-constrained digital microfluidic arrays," in Proc. Design, Autom. and Test in Eur. (DATE) Conf., 2007, pp. 552-557.

[61] T. Xu and K. Chakrabarty, "Broadcast electrode-addressing for pin-constrained multi-functional digital microfluidic biochips," in Proc. IEEE/ACM Design Autom. Conf., 2008, pp. 173-178.

[62] T. Xu and K. Chakrabarty, "Design-for-testability for digital microfluidic biochips," in Proc. IEEE VLSI Test Symp., 2009, pp. 309-314.

[63] P.-H. Yuh et al., "Placement of defect-tolerant digital microfluidic biochips using the T-tree formulation," ACM J. Emerging Tech. Computing Syts., vol. 3, pp. 13.1-13.32, 2007.

[64] P.-H. Yuh et al., "BioRoute: A network flow based routing algorithm for digital microfluidic biochips," in Proc. ICCAD, 2007, pp. 752-757.

[65] P.-H. Yuh et al., "A progressive-ILP based routing algorithm for crossreferencing biochips," in Proc. DAC, 2008, pp. 284-289.

[66] M. Cho and D. Z. Pan, "A high-performance droplet router for digital microfluidic biochips," in Proc. Int. Symp. on Physical Design (ISPD), 2008.

[67] S.-K. Fan, C. Hashi, and C.-J. Kim, "Manipulation of multiple droplets on $\mathrm{N} \times \mathrm{M}$ grid by cross-reference EWOD driving scheme and pressurecontact packaging," in Proc. MEMS, 2003, pp. 694-697.
[68] Y. Zhao, T. Xu, and K. Chakrabarty, "Built-in self-test and fault diagnosis for lab-on-chip using digital microfluidic logic gates," in Proc. IEEE Int. Test Conf., 2008.

[69] Y. Zhao and K. Chakrabarty, "Cross-contamination avoidance for droplet routing in digital microfluidic biochips," in Proc. IEEE/ACM Design, Autom. and Test in Eur. Conf., 2009, pp. 1290-1295.

[70] Global In Vitro Diagnosis Market Analysis PRLog Free Press Release [Online]. Available: http://www.prlog.org/10080477-global-in-vitrodiagnostic-market-analysis.html

[71] World Malaria Day 2009: Key Figures [Online]. Available: http://www. rollbackmalaria.org/worldmalariaday/keyfigures

[72] H. Yang, V. N. Luk, M. Abelgawad, I. Barbulovic-Nad, and A. R. Wheeler, "A world-to-chip interface for digital microfluidics," Anal. Chem., vol. 81, pp. 1061-1067, 2009.

[73] L. Luan, R. D. Evans, N. M. Jokerst, and R. B. Fair, "Integrated optical sensor in a digital microfluidic platform," IEEE Sensors J., vol. 8, pp. 628-635, 2008.

[74] J. H. Song, R. Evans, Y.-Y. Lin, B.-N. Hsu, and R. B. Fair, "A scaling model for electrowetting-on-dielectric microfluidic actuators," Microfluidics and Nanofluidics, vol. 7, pp. 75-89, 2009.

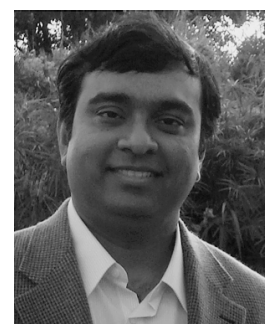

Krishnendu Chakrabarty (S'91-M'92-SM'00F'08) received the B. Tech. degree from the Indian Institute of Technology, Kharagpur, India, in 1990, and the M.S.E. and Ph.D. degrees from the University of Michigan, Ann Arbor, in 1992 and 1995, respectively.

$\mathrm{He}$ is currently Professor of Electrical and Computer Engineering at Duke University, Durham, NC. $\mathrm{He}$ is also a member of the Chair Professor Group (honorary position) in Software Theory at the School of Software, Tsinghua University, Beijing, China. His current research projects include: testing and design-for-testability of integrated circuits; digital microfluidics and biochips, circuits and systems based on DNA self-assembly, and wireless sensor networks. He has authored nine books on these topics (including two in press), published over 320 papers in journals and refereed conference proceedings, and given over 130 invited, keynote, and plenary talks.

Prof. Chakrabarty is a recipient of the National Science Foundation Early Faculty (CAREER) award, the Office of Naval Research Young Investigator award, the Humboldt Research Fellowship from the Alexander von Humboldt Foundation, Germany, and several best papers awards at IEEE conferences. $\mathrm{He}$ is a Golden Core Member of the IEEE Computer Society, and a Distinguished Engineer of ACM. He is a 2009 Invitational Fellow of the Japan Society for the Promotion of Science (JSPS). He is recipient of the 2008 Duke University Graduate School Dean's Award for excellence in mentoring. He served as a Distinguished Visitor of the IEEE Computer Society during 2005-2007, and as a Distinguished Lecturer of the IEEE Circuits and Systems Society during 2006-2007. Currently he serves as an ACM Distinguished Speaker and a Distinguished Visitor of the IEEE Computer for 2010-2012. He is an Associate Editor of IEEE TRANSACTIONS ON COMPUTER-AIDED DESIGN OF INTEGRATEd CiRcuits AND SySTEMS, IEEE TRANSACTIONS ON VERY LaRge SCALE INTEGRATED (VLSI) SyStems, and the IEEE TRANSACTIONS ON BIOMEDICAL CIRCUITS AND SYSTEMS. He also serves as an Editor of the Journal of Electronic Testing: Theory and Applications (JETTA). He is the Editor-in-Chief for IEEE Design \& Test of Computers, and the Editor-in-Chief for ACM Journal on Emerging Technologies in Computing Systems. 